\title{
HPMC Capsules: Current Status and Future Prospects
}

\author{
Moawia M. Al-Tabakha \\ College of Pharmacy, Al Ain University of Science and Technology, Al-Ain, U.A.E. \\ Received, July 13, 2010; Revised, September 20, 2010; Accepted, October 11, 2010; October 13, 2010
}

\begin{abstract}
Hydroxypropyl methylcellulose (HPMC) is employed for a wide variety of pharmaceutical and food preparations. Its applications as viscolizing agent (thickening agent), coating polymer, bioadhesive, in solid dispersion to enhance solubility, binder in the process of granulation and in modified release formulations have been well documented. One other notable use is in the production of capsule shells, replacing the animal derived gelatin in conventional two-piece capsules. The aim of this review is to systemically survey published literature on the HPMC use in capsule shells and resolve questions regarding their suitability as a replacement for hard gelatin capsules. Future refinements in the production and filling of HPMC capsule shells and improvement in their in vivo/in vitro dissolution would ensure their superiority over hard gelatin capsules.
\end{abstract}

\section{INTRODUCTION}

Hydroxypropyl methylcellulose (HPMC), now commonly known as hypromellose, is produced by synthetic modification of the naturally occurring polymer cellulose and is considered safe for normal consumption in humans (1). The material have been used and experimented as viscolizing agent i.e. thickening agent $(2,3)$, in controlled release systems (4) and as a coating polymer (5), as a bioadhesive (6), in solid dispersion to enhance drug solubility (7) and as a binder (8). In the USP30-NF25 (9), the excipient is listed under coating agent, suspending and/or viscosity-increasing agent and tablet binder. The material is described as a white to slightly offwhite powder or granules, hygroscopic after drying, practically insoluble in hot water, in acetone, in dehydrated ethanol and in chloroform, but dissolves in cold water giving a colloidal solution owing to the reversible thermal gelation property. HPMC is available in different substitution type with limits on methoxy and hydroxypropoxy groups. These groups influence many of the HPMC properties such as gelation temperature, viscosity, flexibility and hydration (10).

In addition to the listed excipient categories, HPMC polymer is now used as shell material for capsules (11). The origin of the word capsule comes from the Latin capsula, which means a small box (12). Pharmaceutically, capsules are either hard (two-piece) or soft (one-piece) and are used to encapsulate pharmaceutical formulations (13). The two-piece capsule is made of a cap-piece that slips over one side open body-piece forming closed cylindrical object (14). Capsules may offer better solid dosage form to tablets for drugs with low compressibility, slow dissolution and bitter tasting. They are also used in clinical studies for blinding purpose. The administration of the capsules is usually orally, but capsules for inhalation (15) such as Spiriva HandiHaler (Boehringer Ingelheim International $\mathrm{GmbH}$ ), vaginal (16) such as GynoDaktarin (Janssen-Cilag) and rectal administrations (17) are all possible.

Despite the fact that most of pharmaceutical capsules available in market are made of gelatin, several HPMC capsules for powdered herbs and dietary supplements have been available in recent years. Many investigational new drugs with HPMC encapsulation are in clinical trials (18). HPMC capsules may offer attractive alternative to gelatin capsules because of its vegetable source. The crosslinking of gelatin and drug incompatibilities and the strict regulations regarding the use of animal derived gelatin requiring the absence of bovine spongiform encephalopathy (BSE)/ transmissible spongiform encephalopathy (TSE) have encouraged the search for gelatin replacement. Religious, cultural and personal issues may affect patients' preference towards the medications presented in capsule dosage forms.

Corresponding Author: Dr. Moawia Al-Tabakha, College of Pharmacy, Al Ain University of Science and Technology, AlAin, U.A.E, Email: sphmaa@hotmail.com 
Vegetarians for example are becoming increasingly aware of the capsule shell materials which also encouraged the companies to search for alternatives. As a result, the first vegetable capsules with the trademark Vegicaps made of HPMC were produced in 1989 by G S Technologies Inc. (now R.P. Scherer Technologies ownership).

The first patent registered for gelatin capsule alternative was in 1950 by H W Murphy of Eli Lilly and Company for methyl cellulose which did not last long in the market because of in vivo disintegration delay. Several attempts were made later to improve disintegration notably by Dow Chemical Company and included the use of HPMC. The production of HPMC capsules are by thermal gelation and a gelling system used to lower thermal gelation temperature of HPMC (19). The production technique remains similar to that of hard gelatin capsules and involves the use of pins dipping into HPMC solution, although the machinery may require some modifications such as the use of heated pins. The HPMC capsules patented are not all the same and differ mainly in whether a gelling system is used and in the type of gelling system. Therefore, there are different types of HPMC capsules which may have different in vitro and in vivo performances among themselves and in comparison to hard gelatin capsules.

Since the introduction of HPMC capsules of different kinds is recent and because these shells in pharmaceutical preparations have not acquired full potential, it is necessary to systemically analyze the published literature in terms of their manufacture, in vitro and in vivo performances and to compare them with the hard gelatin capsules. This will help pharmaceutical companies to decide on whether to adventure into using HPMC capsules for their new formulations or use conventional capsules that have enjoyed long successful history.

Searching in the published literature were by using Google (web, scholar and books) at www.google.com, United States Patent and Trademark Office website (www.uspto.gov) and the European Patent Office (www.epo.org). The main search was conducted using Pubmed at http://www.ncbi.nlm.nih.gov/pubmed which utilizes Medline. The later comprises the major component of Pubmed. Searching were made using the terms "HPMC capsule", "hydroxypropyl methylcellulose capsule', "hypromellose capsule", "cellulose capsule", "vegetable capsule", "non-animal capsule" and "hard gelatin capsule". The vast results were filtered by title to include relevant information for the purpose of this review with focus on publications over the last 10 years.

\section{Hard Capsules Production Issues}

\section{HPMC Capsules Manufacture and Types}

Information regarding the empty HPMC capsules and their manufacturer are listed in table 1. The results from the search were filtered to use information regarding the two-piece capsules (hard capsules) only, since there are the soft capsules such as Vegicaps Soft (Catalent Pharma Solutions), HPMC based soft capsules, which are available as alternative to soft gelatin capsules.

Hard gelatin and HPMC capsules are manufactured using similar equipments developed by Eli Lilly (20).

\begin{tabular}{cccc}
\hline \multicolumn{2}{c}{ Table 1. Information on the empty HPMC capsules and their manufacturers. } & & \\
\hline Capsule Shell Brand Name & Manufacturer & Registered Year in USA & Gelling Aid \\
\hline Quali-V & Shionogi Qualicaps & July, 2002 & Carrageenan \\
Vcaps Plus & Capsugel (A division of Pfizer) & - & None \\
Vcaps & Capsugel (A division of Pfizer) & April, 2003 & Gellan gum \\
VegiCaps & G S Technologies Inc. (now R.P. & May, 1989 & None \\
Scherer Technologies ownership) & & Pectin and glycerin \\
Embo Caps -Vg & Suheung Capsule Co., Ltd & - & None \\
Capstech's HPMC Capsule & Baotou Capstech Co., Ltd & - & Carrageenan \\
Natural Plant Capsule & Zhejiang LinFeng Capsules Co. Ltd. & - & \\
\hline
\end{tabular}


In hard gelatin capsule manufacturing, pins (molds for making the capsules) at $22^{\circ} \mathrm{C}$ are dipped in a dip pan or pot that holds a fixed quantity of gelatin at a constant temperature, between $45^{\circ}$ and $55^{\circ} \mathrm{C}$. The level of solution is maintained automatically by a feed from the holding hopper. Once the molds are dipped a film will be formed on them by gelling since they are at lower temperature. The slowly withdrawn pins from the dipping pan are rotated to maintain uniform film thickness, where they are passed through a series of drying kilns at controlled temperature and humidity. The dried films (shells) are stripped of the pins, cut to the correct length and the two pieces (cap and body) are joined together. The pins are then cleaned and lubricated to start the next cycle.

The manufacture of HPMC based capsules necessitates some modification to the molding machine or to the formulation of the shell materials. HPMC gelling from solution occurs when the temperature is raised while it is converted to its original solution as the temperature is lowered, unlike gelatin solution. This means that the pins immersed in the dip pan containing the HPMC solution must be of higher temperature $\left(70^{\circ} \mathrm{C}\right)$ in order for the film to be formed. To avoid liquefaction of the films formed on the pins, the temperature of the pins must be further maintained post-dip to facilitate gelation until the films dry out in the kilns (21-24).

Because HPMC shell walls are much weaker than gelatin made shells, removal of the capsule from the pins and subsequent handling and filling are in jeopardy. To overcome these problems, three approaches were adapted. These approaches were to use a stripper jaw with depressions on the inner surface, increase the formed HPMC film thickness and the use of gelling agents. The following gelling agents were experimented: tamarind seed polysaccharide, carrageenan, pectin, curdlan, gellan gum and furcellaran.

U.S. Pat. Nos. 5,264,223 and 5,431,917 registered for Yamamoto et al. $(25,26)$ of Japan Elanco Company, Ltd. (Osaka, JP) claiming that capsules can be produced by the use of HPMC with the gelatinizing agent such as carrageenan $\left(\mathrm{HPMC}_{\text {carr }}\right)$ and auxiliary for gelation is a watersoluble compound containing potassium ion. The production of such capsules were claimed to occur under similar temperature setting as that of gelatin capsules. Shionogi Qualicaps Co. (Japan) was able to produce $\mathrm{HPMC}_{\text {carr }}$ capsule using the standard machinery for the hard gelatin capsule by using HPMC gelling system containing carrageenan as a gelling aid (kappa- and iota- carrageenans are preferred) and potassium chloride as gelation promoter. The company has a Quali-V registered trademark. European patent EP0592130 claims that HPMC with higher whiteness, lower equilibrium moisture content and better film properties and compatibility with drugs could be produced by exposing the materials to ultraviolet light in the wavelength range of at least $200 \mathrm{~nm}$ (27). The claim indicates that at the wavelength $253.7 \mathrm{~nm}$, the preferred conditions for ultraviolet radiation are a spacing of about $10 \mathrm{~cm}$ for about 10 hours.

Yang (28) of Suheung Capsule Co., Ltd., a Korean based company, had patented cellulose capsules using mixed solution of pectin and glycerin. The steps involved in the manufacture are to add the mixed solution of pectin and glycerin to the HPMC solution followed by the addition of small amount of glacial acetic acid, calcium gluconate, and sucrose fatty acid ester.

An invention of Warner-Lambert Company (now with Capsugel that later became part of Pfizer) (29) have documented the preparation of HPMC capsules with hydrocolloids such as gellan gum $\left(\mathrm{HPMC}_{\text {gell }}\right)$ and sequestering agents (such as ethylenediaminetetraacetic acid, sodium citrate, citric acid and their combinations). Gellan gum is a water-soluble polysaccharide produced by the bacteria Sphingomonas elodea. $5 \%$ of the capsule shell materials comprised of approximately equal proportions of both the hydrocolloid and the sequestering agent. The claim shows that these capsules would have films that are less brittle (unlike those produced with carrageenans), no poor disintegration in vivo and the film transparency is retained.

It is worth mentioning that most of the companies producing HPMC capsules such as Capsugel of Pfizer and Shionogi Qualicaps are also producing the standard gelatin capsules.

\section{Marketed Products}

The HPMC capsule shells have found popularity for their use with nutraceuticals and over-the-counter (OTC) formulations. Using Google web search, many nutritional products were found. Only few examples of those with sufficient information obtained are given in table 2 . 


\begin{tabular}{|c|c|c|}
\hline Product & Nature of the Formulation & Manufacturing Company \\
\hline Damiana Herb 300mg & Pure powdered herbs (Damiana turnera aphrodisiaca) & Bio-Health Ltd., UK \\
\hline $\begin{array}{l}\text { Thera Veda's Ajay- Allergy } \\
\text { Support Formula }\end{array}$ & Vegetable extracts and powders & Organix South, USA \\
\hline Natren Life Start 2 & Bacteria, vitamin $\mathrm{C}$, potato powders and whole goat milk & NATREN, Inc., USA \\
\hline Coloclear (in VegiCap) & Flax seeds, slippery elm and other herbs & Higher Nature Ltd., UK \\
\hline Jarro-Dophilus EPS & 8 probiotic species and ascorbic acid & Jarrow Formulas, USA \\
\hline Culturelle HS Capsules & $\begin{array}{l}80 \text { mg lactobacillus GG (L. rhamnosus GG) Vegetarian } \\
\text { Formula }\end{array}$ & Kirkman Labs, USA \\
\hline $\begin{array}{l}\text { Align Daily Probiotic } \\
\text { Supplement Capsules }\end{array}$ & Bifidobacterium infantis & Procter and Gamble, USA \\
\hline Sportlegs Supplement & Vitamin $\mathrm{D}$, calcium and magnesium & Sportlegs, USA \\
\hline $\begin{array}{l}\text { Planetary Herbals Cinnamon } \\
\text { Extract }\end{array}$ & $\begin{array}{l}\text { Cinnamomum aromaticum } 300 \mathrm{mg} \text {, } \\
\text { bark extract } 10: 1 \text { yielding } 8 \% \text { flavonoids, } \\
\text { cinnamomum aromaticum bark } 100 \mathrm{mg}\end{array}$ & Planetary Herbals, USA \\
\hline Ex-Tox II & $\begin{array}{l}\text { Folic acid, cilantro powder (leaf), ethylenediamine tetra- } \\
\text { acetic acid, N-Acetyl L-cysteine, fulvic (humic) acid, R- } \\
\text { lipoic acid (K-RALA), L-methionine }\end{array}$ & Progressive Labs, USA \\
\hline
\end{tabular}

\section{Capsule Size Information}

HPMC capsules are available in similar physical dimensions of sizes and shell weights as that of hard gelatin capsules. Gelatin capsules are the main stream in the production for most capsule manufacturing companies and because their production has been standardized over long period, they are available in wider range of sizes. For example Capsugel company (division of Pfizer) produces Coni-Snap hard gelatin capsules in the standard sizes from 000 to 5 with elongated sizes (have capacities approximately $10 \%$ more than the standard ones) for capsules $00,0,1$ and 2, while the same company produces Vcaps Plus HPMC capsules with sizes from 00 to 4 with elongated capsules for size 0 . Quali-V capsules are available in sizes from size 0 to 4 with elongated size 0 only and the empty shells weights varied by $\pm 10 \%$ according to the Qualicaps Group company website, but not exceeding $8 \%$ for Vcaps Plus according to the Capsugel company website. If the variations in the capsule shell weights are large, this may result in several filled capsules being rejected from the batch during weight sorting, even though the filled weights are accurate.

The cross sectional part of the capsule joints has been evaluated under electron microscope for three types of capsule shells (18). The examination intended to measure the maximum observed gap between the body and the cap. It was found that the largest maximum gap is for Quali-V capsules $(132.14 \mu \mathrm{m})$, while Vcaps Plus capsules showed slightly larger gap than that of hard gelatin capsules
Coni-Snap (88.77 and $66.86 \mu \mathrm{m}$ respectively). The benefit of this decrease in gap clearance with Vcaps Plus compared to Quali-V meant improvement in the powder leakage quality attribute and decrease in the rejection rates. It is worth mentioning that this was reported by researchers from Capsugel Company (division of Pfizer), the producer of Vcaps Plus.

The relationships between wet film dimensions, dip sequences, and the physicochemical properties of the dip solutions in the manufacture of hard-shell capsules were studied (30). In the dipping process for making hard-shell HPMC capsules, the effects of solution concentration is more important than pin temperature. It is however difficult to predict wet film thickness in a hot-pin, cold solution dipping process.

\section{Mechanical Strength}

In a test examining the effect of humidity on the mechanical properties of both HPMC and gelatin capsules, it was found that both types of capsules softened, especially above $60 \%$ of relative humidity, with gelatin capsules exhibiting in general higher stiffness and hardness values compared to the HPMC capsules (31). In another study (32) it was found that at ambient conditions, capsules made from gelatin were harder and stronger but less elastic compared with HPMC counterparts.

The mechanical stress applied to the empty Vcaps Plus HPMC capsules and empty Coni-Snap hard gelatin capsules $(n=50)$ were evaluated by 
dropping $100 \mathrm{~g}$ weight from $8 \mathrm{~cm}$ height on each capsule following storage at different relative humidity of approximately $5-65 \%$ for one week (18). The resistance to breakage was similar for both types of capsules at higher relative humidity but at lower humidity the Vcaps Plus capsules were less affected compared to Coni-Snap capsules, as the later exhibiting an increase in the breakage rates because of the loss of water responsible for the hard gelatin capsules elasticity. Further tests on the capsules to evaluate their machineability under different filling machines at various speeds, during packaging and transportation were carried out. With the different filling machines, the rejection was lowest with hard gelatin capsules (Coni-Snap) compared to other HPMC capsule types (Vcaps Plus and Quali-V) and this was attributed mainly to problems in the closing station. The blistering process and carding the blisters containing Vcaps Plus with placebo powder have shown no visual powder leaking. While the Quali-V capsules showed 6\% leak rate around the joint of capsule body and cap, Vcaps Plus and Coni-Snap showed none in a simulated transport test.

\section{Effects of Ambient Conditions}

The use of capsules as means for rapid disintegration in the oral cavity was experimented (33). One approach was to cause the capsules to become brittle in order to brake rapidly in the oral cavity by subjecting them to low humidity. While this approach was successful for hard gelatin capsules, HPMC capsules remained flexible, even at low moisture content. Short term stability studies (heating at different temperature for $24 \mathrm{hrs}$ ) on both Vcaps Plus and Coni-Snap found that overall, the HPMC shell exhibits a significantly better short term stability at high temperature than hard gelatin capsules on visual test, disintegration and dissolution, as well as mechanical property assessment (18). When they were stored at different relative humidity $(\mathrm{RH})$, the HPMC capsules exhibited lower moisture contents compared to gelatin capsules (e.g. $6 \%$ and $14 \%$ respectively at $50 \% \mathrm{RH})$ that have shown to be more hygroscopic. Based on the previous study, the specifications for moisture content are $2-7 \%$ for the HPMC shell corresponding to RH $10-60 \%$ and $13-16 \%$ for gelatin capsules corresponding to storage at RH 35$65 \%$. Preliminary results from the effect of irradiation (beta or gamma) on both HPMC and gelatin capsules in air indicated their suitability for ionizing radiation sterilization (34).

\section{IN VITRO Disintegration and Dissolution}

Because the USP only mentions the testing of gelatin capsules, Donauer and Löbenberg (35) have called in a min review the USP to specify how to carry out the disintegration test with HPMC capsules. That is because the dissolution behaviors of HPMC and gelatin capsules have to be different in dissolution media. Moreover, HPMC capsules are not all the same as they may or may not contain a gelling agent and the gelling agents used are not all the same.

The shell dissolution properties of ordinary gelatin hard capsules, gelatin/PEG capsules and $\mathrm{HPMC}_{\text {carr }}$ capsules were compared independent of their capsule content (19). Different dissolution media and storage conditions were used. The capsule shells disintegration/dissolution time was determined as the time for enough parts of the suspended capsule to dissolve, permitting steel ball bearing filled into the capsule to fall free. Capsules were placed in media of different temperature (between $10^{\circ}$ and $55^{\circ} \mathrm{C}$ ) in order to simulate taking the capsules with cold, warm or hot drinks. The dissolution media in the glass beaker at different temperatures were brought back to $37^{\circ} \mathrm{C}$ with the controlled temperature of the surrounding water bath. Gelatin and gelatin/PEG capsules disintegrated rapidly and faster than the $\mathrm{HPMC}$ carr capsules in the different media following storage at different conditions when tested at temperature $\geq$ $37^{\circ}$ C. This delay in the HPMC capsule disintegration was especially notable in mixed phosphate buffer of $\mathrm{pH} 6.8$. The delay at $\mathrm{pH} 6.8$ is inherent for the HPMC shells (36). In water at $37^{\circ}$ $\mathrm{C}$ following storage at ambient room conditions $\left(19 \pm 1{ }^{\circ} \mathrm{C}, 35-40 \%\right.$ relative humidity of the air) $\mathrm{HPMC}_{\text {carr }}$ capsules disintegrated in approximately 4 minutes whereas gelatin and gelatin/PEG capsules disintegrated in approximately 1 minute. Gelatin capsules dissolution times are dependent upon temperature and generally do not dissolve at temperatures below $30{ }^{\circ} \mathrm{C}$, however, their dissolution was rapid as temperature increased from $30^{\circ}$ to $55^{\circ} \mathrm{C}$ suggesting that gelatin capsules are better be taken with warm water.

The influence of the composition of test fluids on dissolution from $\mathrm{HPMC}_{\text {carr }}$ capsules (Quali-V) in comparison to the hard gelatin capsule was studied 
(37). The results were in agreement with another study (19) showing significant retarding effect of potassium and/or calcium ions in the dissolution medium, while the effect of $\mathrm{pH}$ was minimal on the acetaminophen (BCS class III) dissolution. Similar effects of dissolution media were also documented when studying the effects of dissolution medium, capsule grade and capsule size on the in vitro rupture time of the capsule shells (38). The results further indicated that the capsules used whether pharmaceutical $\mathrm{HPMC}_{\text {carr }}$ capsules, nutritional $\mathrm{HPMC}_{\text {carr }}$ capsules or gelatin capsules all rupture in different times in vitro with gelatin capsules being the fastest. Stein and Bindra (39) who used HPMC capsules from Shionogi for their formulations found that in an acidic $\mathrm{pH}(0.1 \mathrm{~N} \mathrm{HCl})$, the dissolution of the capsules formulations were retarded in comparison to hard gelatin capsules at earlier times and therefore delaying the time of complete drug dissolution.

Size 0 hard HPMC (Shionogi Qualicaps) and gelatin (Coni-Snap, Capsugel) capsules were tested for ibuprofen (BCS class II) release in tribasic sodium phosphate buffer $\left(\mathrm{pH} 7.2,900 \mathrm{ml}\right.$ at $\left.37^{\circ} \mathrm{C}\right)$ with formulation containing release modifiers (powdered HPMCs grades as diluents). As the medium contained no potassium (apparently to prevent its influence on the dissolution from HPMC capsules), both types of capsules showed similar dissolution profiles (40). However, it is apparent that such formulations influence their own release, irrespective of the capsule shell rupture time, therefore not sharp indicative of the capsule rupture time in the dissolution medium.

Honkanen (41) showed that when ibuprofen formulation in $\mathrm{HPMC}_{\text {carr }}$ capsules tested for drug release in a neutral potassium phosphate buffer, it was incomplete and highly variable compared with the gelatin capsules and attributed this to the presence of potassium ions $\left(\mathrm{K}^{+}\right)$in the dissolution medium that causes the capsule shell to form a membrane around the filling. Because the gut concentration of potassium is low, she justified the change of dissolution medium to neutral tribasic sodium phosphate which resulted in complete and less variable drug release. In this medium $100 \%$ of the drug was released for both types of capsule within 15-20 minutes, however, there was a lag time of approximately 4 minutes before the drug release from $\mathrm{HPMC}_{\text {carr }}$ capsules, unlike gelatin capsules in which the release was immediate.

Similar results were obtained when testing the dissolution of $\mathrm{HPMC}_{\text {gell }}$ capsule shells that are filled with ibuprofen in comparison to hard gelatin capsules at $\mathrm{pH} 7.2$ using potassium phosphate and TRIS buffers (42). It was found that the presence of $\mathrm{K}^{+}$cations retards $\mathrm{HPMC}_{\text {gell }}$ capsule opening with the drug dissolution approaching $60 \%$ after 60 minutes compared to approximately $95 \%$ at 10 minutes for hard gelatin capsules. It was also reported that for acetaminophen (see Figure 1), the release delay was lessened when sodium ions were present instead of potassium in phosphate buffer at $\mathrm{pH} 7.2$ or in acetate buffer at $\mathrm{pH} 4.5$ and that the $\mathrm{HPMC}_{\text {gell }}$ capsules failed to rupture with very little drug release when the medium was acidic $(0.1 \mathrm{~N}$ $\mathrm{HCl}, \mathrm{pH}=1.2$ ). The authors explained the hindrance of the $\mathrm{HPMC}_{\text {gell }}$ capsule dissolution in potassium phosphate buffer as due to the monovalent cations binding to the surface of individual helices of gellan, thus lowering their charge-density and reducing the electrostatic barrier to aggregation and hence solubility is reduced. They proposed that sodium ions do not efficiently bind as potassium ions and therefore disruption will be faster.

They also explained that unlike the sulfate groups in carrageenan gelling system, the carboxyl groups of gellan gum, have a much higher $\mathrm{pKa}$ resulting in uncharged (-COOH) form at low $\mathrm{pH}$. This elimination of electrostatic repulsion between helices makes gellan less soluble at $\mathrm{pH}$ 1.2. HPMC solubility on the other hand is independent of $\mathrm{pH}$ (10).

\section{Cross-Linking of the Hard Capsules}

Several studies have shown the detrimental effect of cross-linking between gelatin proteins on the solubility of the gelatin made capsules $(18,43-45)$ which may affect drug's bioavailability. Experiments exposing HPMC capsules to similar conditions under which hard gelatin shells undergone cross-linking have indicated the absence of such reaction (44). Cross-linking occurs because of the chemical interactions between gelatin and aldehydes, such as formaldehyde and also when the capsules are stored under stressed conditions of temperature and humidity. Attempts to reduce cross-linking include the modification of excepients used with drugs. 


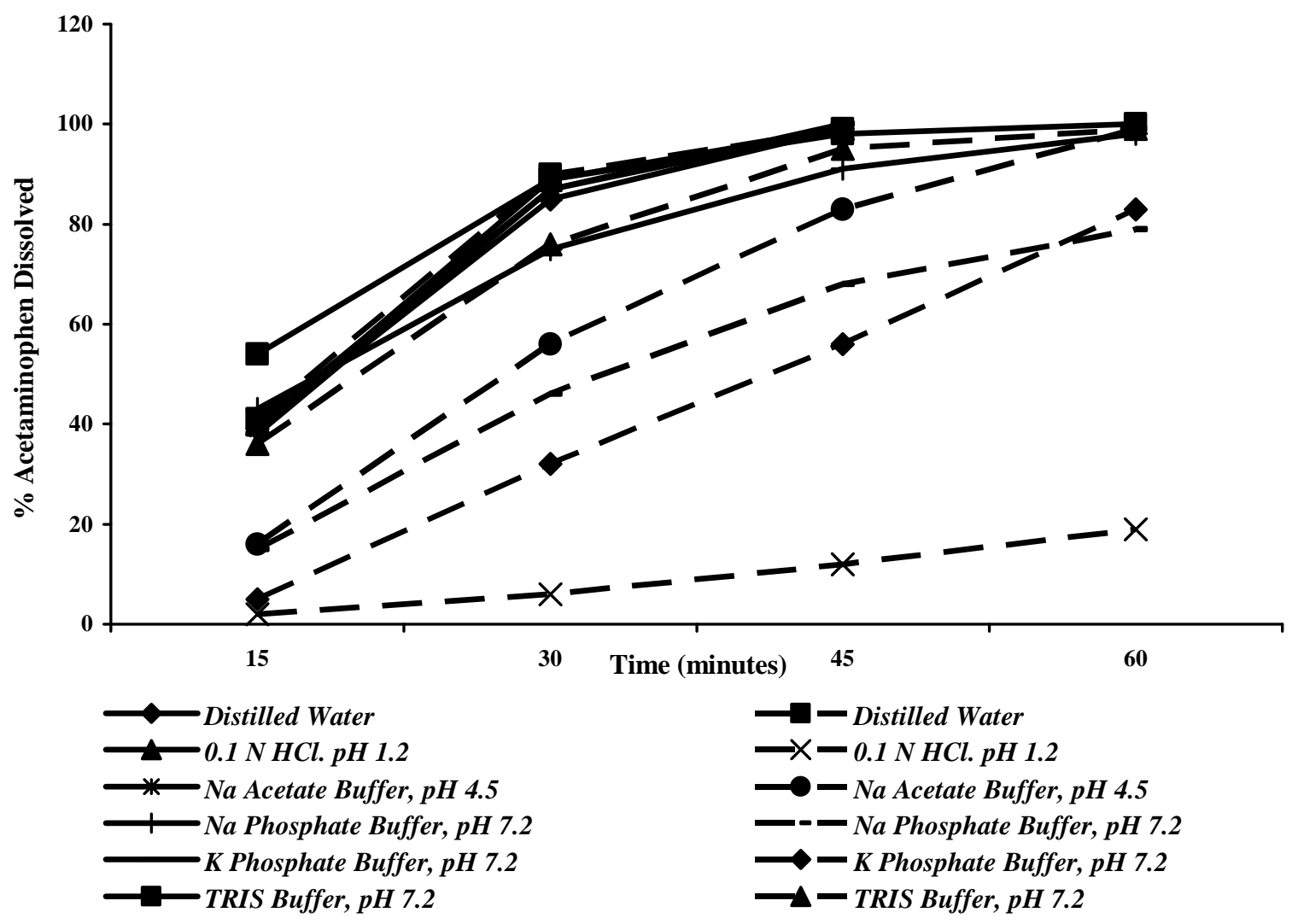

Figure 1. The dissolution of acetaminophen from HPMC capsules (dashed lines) and gelatin capsules (continuous lines) in different dissolution media $(\mathrm{n}=6)$. Graph generated from Cole et al. published data (42).

For example a combination of glycine and citric acid in some drug formulations to prevent crosslinking in hard gelatin capsules has been used (46). PEGs used as a fill material in hard gelatin capsules may be responsible for cross-linking of gelatin following its oxidation. This has shown to be minimized when butylated hydroxyanisole (antioxidant) together with water were used in the formulation even when stored at $50{ }^{\circ} \mathrm{C}$ for three months (44).

\section{Capsules of Dry Powders for Inhalation}

Capsules were first used for dry powder inhalers 40 years ago with the introduction of Spinhlaer of Fison (now with Sanofi-Aventis) which uses two pins to puncture the capsules and deliver cromolyn sodium (sodium cromoglycate). HPMC capsules were recommended for use in unit-dose inhaler in comparison to the hard gelatin capsules, especially for hygroscopic materials (47). This is because the gelatin capsules have relatively high moisture content (13-16\%) in comparison to 4-6\% for HPMC capsules; therefore an interaction of the powdered materials with the gelatin capsule would retain much of the powder adhering to the inner surfaces of capsules resulting in much of the dose failing to leave the device (48). In fact one of promoting strategies for HPMC capsules is their suitability for hygroscopic materials.

Devices such as Spinhlaer, the first marketed dry powder inhaler, and Foradil inhaler (Novartis) rely on piercing the loaded capsule and withdrawing the powdered aerosol by inhalation. The holes created by piercing of the capsule were found to be different for HPMC and gelatin capsules and dependant on the relative humidity (49). In low humidity (below 10\%) the gelatin capsule shell becomes brittle and this could cause the pierced parts of the capsule to detach, which may be inhaled causing irritation to the throat and lungs. 


\section{Capsule Coating}

Enteric and colonic delivery of HPMC capsules were claimed (50) by using coating materials of different $\mathrm{pH}$ solubility (at 5.5 and above and at 7 and above for enteric and colonic delivery respectively). The US patent describes how aqueous dispersions of materials such as cellulose acetate trimellitiate, hydroxypropylmethyl cellulose phthalate, polyvinyl acetate phthalate, shellac, copolymer of methacrylic acid and methylmethacrylate, azopolymers, disulphide polymers and amylase are sprayed on the filled HPMC capsules when placed in Accela-Cota 10 in order to achieve targeted delivery.

To avoid the lengthy and expensive sealing step required using the conventional capsule coating procedure and to prepare enteric-coated capsules for the use in retail or hospital pharmacy or R\&D sections of pharmaceutical industry, the caps and bodies of $\mathrm{HPMC}_{\text {gell }}$ capsules size 00 (Vcaps, Capsugel) were coated separately prior to filling in a fluid bed apparatus (GPCG-1, Glatt) with Eudragit L30D-55 or Eudragit FS 30 D (Röhm), Aqoat AS-HF (Shin-Etsu) and Sureteric (Colorcon), using an optimised coating process (51). This has resulted in effective protection of drug release from the capsules in $0.1 \mathrm{HCl}$ after $2 \mathrm{hr}$.

The comparison between the coating of gelatin capsules and HPMC capsules showed that the later coating was straight forward, while gelatin capsules were not suitable for direct coating when Eudragit L and S 12.5 (acrylic polymers) was used because of insufficient film adhesion to the smooth capsule surface and the brittleness of formed films (52). Because HPMC capsule shell surface is rougher compared to gelatin capsules as examined by scanning electron microscope, this may provide good adhesion to the coating (53).

\section{IN VIVO Evaluation of the Hard Capsules}

\section{Oesophageal Sticking Tendency}

Perkins and colleagues (54) have compared the oesophageal transit of radiolabelled enteric coated tablets with similar sized and shaped gelatin capsules when administered with $50 \mathrm{ml}$ of water while sitting on two separate occasions, using a population of elderly healthy volunteers $(n=23)$. The capsules showed tendency for longer holdups in the oesophagus (20.9 s) compared to enteric coated tablets $(4.3 \mathrm{~s})$.
HPMC as a bioadhesive material was reviewed and experimented by researchers $(55,56)$. It is therefore expected that an increase in the oesophageal residence time would occur before reaching stomach when HPMC capsule is used as a result of HPMC sticking. This tendency to stick to isolated porcine oesophageal preparation was evaluated (40). It was found that $\mathrm{HPMC}_{\text {carr }}$ capsules detached more easily compared to gelatin capsules $(\mathrm{P}<0.001)$. Although their earlier findings indicated easier detachment of HPMC capsules from isolated porcine oesophagus, Honkanen et al. (41) have recommended that both HPMC capsules as well as gelatin capsules be taken with a sufficient amount of water (150-200 ml) in an upright position and maintaining the upright position for several minutes since they found that HPMC capsules had a tendency to attach to the oesophagus. It has been shown that the in vitro porcine esophageal model is not correlated to esophageal transit in man and the recommendation was to use gamma scintigraphy to evaluate esophageal transit in man (57). In general, to avoid oesophagus entrapment of solid dosage forms it is advocated that they should be taken in upright body position with at least $50 \mathrm{~mL}$ of water to minimize entrapment in the (58).

The comparison of $\mathrm{HPMC}_{\text {gell }}$ capsules with conventional hard gelatin capsules showed no significant differences between the two with most capsules having transit time $<20 \mathrm{~S}$ (42). In that study few of both gelatin and HPMC capsules administered had oesophageal hold-up up to $90 \mathrm{~s}$ when administered to eleven healthy subjects. In contrast to this, it was found that the transit times for $\mathrm{HPMC}_{\text {carr }}$ capsules (QUALI-V, Qualicaps) and gelatin capsules (Qualicaps) of size 0, filled with lactose-based mixture, were similar and rapid ranging from 10-20 $\mathrm{s}$ with no prolonged oesophageal hold-ups observed with any subject (59). The test was carried out by administering both capsules simultaneously with $180 \mathrm{ml}$ water to eight healthy male subjects following an overnight fast.

\section{In Vivo Disintegration and Dissolution}

Two prolonged release, radiolabelled formulations, containing different viscosity grades of HPMC powder (HPMC K100 and HPMC K4M) filed in $\mathrm{HPMC}_{\text {carr }}$ capsules size 0 from Shionogi Qualicaps were tested in 6 healthy volunteers with one week wash-out period between the two administrations to examine the fate of the capsules in the GIT (60). 
The initial disintegration times for the capsules were measured as the midpoint of the time interval between the last image of the capsule with clear outlines and visually undetectable spreading of the radioactivity and the time of first detection of spreading radiation. It was found that in 4 occasions out of 12, the capsules were lodged in the oesophagus for 22-143 $\mathrm{min}$. For the two formulations the initial disintegration time ranged from 33 to 75 minutes with no significant difference at the 5\% level (Figure 2). All of the administered capsules started the disintegration in the small intestine except for two which started in the oesophagus region at 75 minutes for each of the two formulations.

In the Tuleu and colleagues study (61) they found that all of the administered uncoated capsules $\mathrm{HPMC}_{\text {carr }}$ capsules (Shinogi Qualicaps) disintegrated within $10 \mathrm{~min}$ in the stomach. The radiolablled capsules were filled with 550-mg dose of 4-aminosalicylic acid. A study conducted to test the disintegration of $\mathrm{HPMC}_{\text {carr }}$ and gelatin capsules in eight healthy male subjects following an overnight fast using QUALI-V and gelatin capsules of size 0 produced by the same company (Qualicaps) (59) agreed with Tuleu et al. (61) results. Both types of capsule were first radiolabled with indium-111 and technetium-99m and then filled with a plug of lactose-based formulation. Both capsules were administered simultaneously to each individual with $180 \mathrm{ml}$ of water. Initial capsule disintegration time was recorded when the scintigraphic image first shows the spread of radioactivity from the 'core' of the capsule. The in vivo disintegration times were not significantly different $(p=0.108$, paired $t$-test $)$ for $\mathrm{HPMC}_{\text {carr }}$ capsules $(9 \pm 2 \mathrm{~min})$ and gelatin capsules $(7 \pm 4 \mathrm{~min})$ with the first showing more consistent behavior as show in Figure 3.

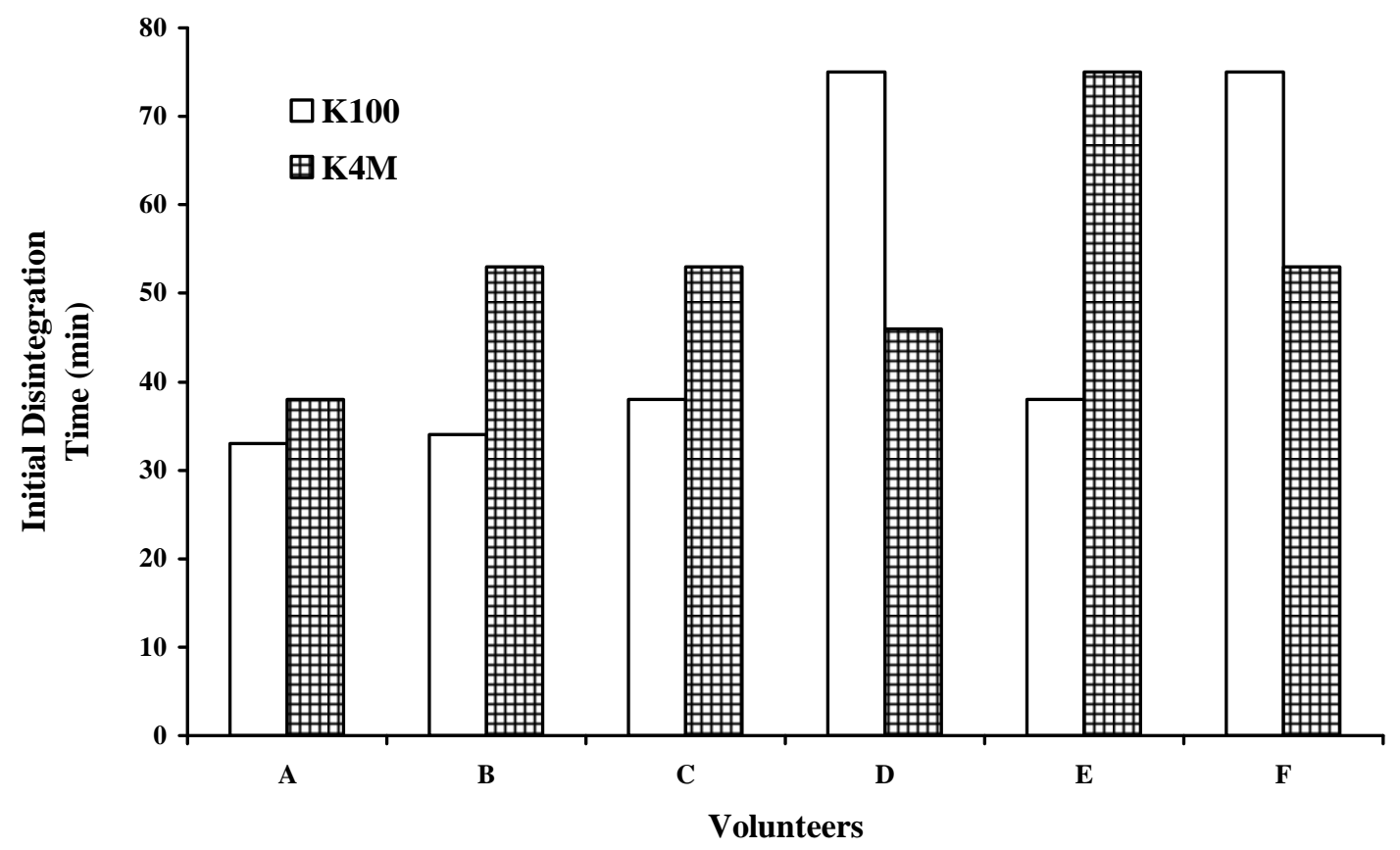

Figure 2. In vivo initial disintegration time (minutes) for the HPMC capsules in 6 healthy volunteers filled with two different prolonged formulations containing different viscosity grades of HPMC powder (HPMC K100 and HPMC K4M). Graph was generated from data published by Honkanen and colleagues (60). 


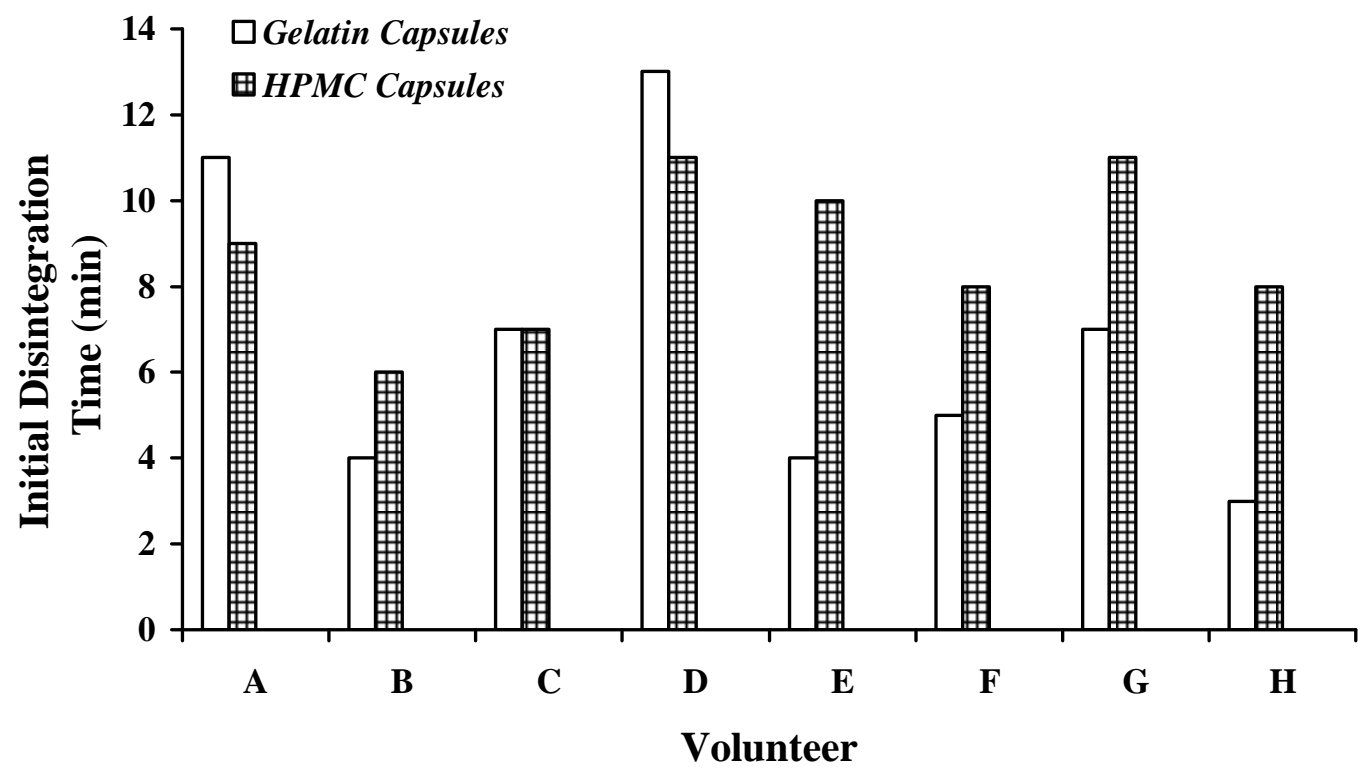

Figure 3. In vivo initial disintegration time ( $\mathrm{min})$ for HPMC and gelatin capsules in 8 healthy volunteers. Graph was generated from data published by Tuleu and colleagues (59).

The studies conducted by Honkanen et al. (60) and Tuleu et. al. (59) were carried out on test subjects of similar characteristics in terms of age and weight and received the test capsules with $180 \mathrm{ml}$ of water following an overnight fasting. Two main differences in the findings of the two studies are the lodging of HPMC capsules in the oesophagus region and the initial disintegration time. The prolonged release formulations used in Honkanen and co-workers study (60) and the fact that after the subjects received the capsules in sitting position were required to lie down may explain the differences observed. The formation of a gelatinous plug by the hydrated hydrophilic polymers (HPMC $\mathrm{K} 100$ and $\mathrm{K} 4 \mathrm{M}$ ) in water may retard the release of the formulation from the capsule shells, independent of the rupture time of the capsule shell. Not only HPMC is a bioadhesive material, but the rougher surfaces of HPMC capsules compared to hard gelatin capsules (53) may also partially explain why HPMC capsules may lodge in the oesophagus region.

Cole and his colleagues (42) found that in both the in vivo fasted and fed state, gelatin capsules disintegrated faster than $\mathrm{HPMC}_{\text {gell }}$ capsules in formulations containing ibuprofen. The results from gelatin capsules agree with that of Tuleu et al. (59), but not for HPMC capsules. The HPMC capsules results, however, agreed with the Honkanen et al. (60) results who used prolonged release formulations for their $\mathrm{HPMC}_{\text {carr }}$ shells. It is possible that poor performance of $\mathrm{HPMC}_{\text {gell }}$ capsules in the acidic environment is attributed to the gelling system used, namely gellan.

\section{Bioavailability Studies}

Tuleu and colleagues (61) investigated using a combined scintigraphic and pharmacokinetic approach in 7 healthy volunteers, the in vivo performance of amylose-ethylcellulose-coated HPMC capsules (size 0, Shinogi Qualicaps) as well as uncoated capsules for the delivery of 4aminosalicylic acid $\mathrm{Na}(550 \mathrm{mg})$ to the colon. The results of $\mathrm{T}_{\max }(29 \min \pm 9)$ and $\%$ absolute bioavailability based on AUC $(118 \pm 41)$ indicated that the uncoated capsule contents were released and absorbed rather completely and rapidly.

Honkanen and her colleagues (40) found that the bioavailability of sustained release ibuprofen formulation administered orally from HPMC capsules was only significantly different from gelatin counterparts when HPMC K100 diluent was 
used as indicated by $\mathrm{T}_{\max }$ which occurred earlier in the case of gelatin capsules $(2.19 \mathrm{~h}$ vs. $3.25 \mathrm{~h}, \mathrm{p}<$ $0.01, \mathrm{n}=8)$. Such significant differences were not observed with the higher viscosity grades of HPMC $\mathrm{K} 4 \mathrm{M}$ and HPMC K15M. It is worth mentioning that as the use of HPMC K100 release modifier resulted in only modest sustained release effects; sharper differences between HPMC and gelatin shells would be expected for immediate release formulations. The previous results of Honkanen et al. (62) for immediate release ibuprofen formulations did not show such differences orally, but there were differences following rectal administration. The lag time $\left(\mathrm{T}_{\mathrm{lag}}\right)$ from $\mathrm{HPMC}_{\text {carr }}$ capsules were higher $(p<0.05, n=8)$ than those for the gelatin capsules.

Four-way crossover experiment were carried out in 11 subjects in fed and fasting states by administering ibuprofen formulations filled in gelatin capsules and $\mathrm{HPMC}_{\text {gell }}$ capsules (42). Scintigraphic and pharmacokinetics evaluations indicated that although the in vivo opening times of $\mathrm{HPMC}_{\text {gell }}$ capsules were longer than gelatin counterparts, the pharmacokinetic parameters $C^{\max }$ and AUC showed no significant difference. There were significant differences in the $\mathrm{T}_{\text {lag }}$ (time before absorption could be detected) for the two capsules whether in the fasted or fed state probably as a result of delayed initial disintegration of the $\mathrm{HPMC}_{\text {gell }}$ capsules.

Pain has been shown to suppress nervus vagus that is responsible for gastrointestinal secretion and motility, therefore impairing the absorption of drugs administered orally resulting from reduced disintegration and dissolution. This would influence drug bioavailability from standard medications, while for rapid release formulations, dissolution and disintegration are independent of the gastrointestinal secretion and motility $(63,64)$. It will be interesting to compare the effect of pain on drug absorption from filled HPMC and gelatin capsules, as any increase in the $\mathrm{T}_{\text {lag }}$ is clearly a disadvantageous in such condition.

\section{IN VITRO-IN VIVO Correlation}

Unlike hard gelatin capsules, HPMC capsules may have low correlation between the in vitro dissolution/disintegration and the in vivo performance. The reason for this was explained on the basis of interaction between the medium and the HPMC capsule gelling systems. It was suggested that dissolution/disintegration testing specifications should be different from that of hard gelatin capsules to reflect in vivo performance. For hard gelatin capsules, for the in vitro testing to correlate with in vivo evaluation, it has been suggested that dissolution experiment is carried out in two stages, one representing gastric medium (pepsin at $\mathrm{pH}$ 1.2) and the other representing the intestinal medium (pancreatin at $\mathrm{pH}$ 7.2) (43). El-Malah and his colleagues (65) indicated that the composition of the dissolution medium influences the disintegration time of the HPMC capsules, however, drug release delay in vitro may not be correlated in vivo.

While the pharmacokinetic results from in vivo oral administration studies of modified release ibuprofen formulations indicated good agreement with in vitro dissolution studies conducted in tribasic sodium phosphate buffer of $\mathrm{pH} 7.2$ (which contains no potassium), rectal administration did not show such correlation (40). It is worth mentioning that interpatient variability in pharmacokinetic parameters was higher for rectal compared to peroral administration.

\section{DISCUSSION}

Several HPMC capsule shells are now available and differ mainly in whether a gelling system is used or not. The gelling system is used mainly so that the manufacturing of the capsule shells can be performed using the same equipments as that of hard gelatin capsules under similar processing conditions. The gelling system used may retard the disintegration/dissolution of HPMC capsules in vitro/in vivo, but does not usually affect product bioavailability. The hindrance of in vitro disintegration/dissolution occurs when cations such as potassium (usually used in the gelling system of HPMC capsules) are present in the media, causing persistence gelling of the capsule shells. Also the in vitro disintegration/dissolution has been documented to decrease in acidic medium for $\mathrm{HPMC}_{\text {gell }}$ capsules.

The use of HPMC for making capsules without a gelling system may reduce problems associated with dissolution/disintegration, however unlike hard gelatin capsules, the disintegration and dissolution of HPMC decreases as temperature increases above $30^{\circ} \mathrm{C}$. Therefore there has been a suggestion to administer hard gelatin capsules with warm drink. HPMC on the other hand is soluble even below $30^{\circ}$ 
$\mathrm{C}$ and as low as $10^{\circ} \mathrm{C}$ and therefore can be taken with cold water.

The machineability of the HPMC does not match that of hard gelatin capsules. Not only, the processing in the manufacture of HPMC shells may need to be altered and/or gelling system added, but also the shell may have reduced strength and much higher rejection when used in filling machines. So, dissolution/disintegration performances and the machineability of the HPMC capsules are probably the major drawback of HPMC capsules.

The main advantage of HPMC capsules over gelatin capsules could be because of their vegetable source which has wider customer acceptance (32). Hindu or Buddhist for example rely on vegetable sources for their nutrition $(66,67)$. Muslim and Jews on the other hand have strict regulations about materials from animal sources and for whom vegetable source is acceptable. To this extent other types of capsules have been produced from nonanimal sources such as NPcaps capsules (Capsugel of Pfizer) which are made from pullulan, a watersoluble polysaccharide produced through a fermentation process.

Fish based gelatin capsules are also available in the market (EMBO CAPS-Fish from Suheung Capsule Co., Ltd). The fish gelatin solution from which the shells are produced contains mixed solution of pectin and glycerin as gelling agent and a small quantity of calcium gluconate, sucrose fatty acid esters, glacial acetic acid as additive (68). These capsules may offer alternative to people with concern from gelatin produced from bovine and/or porcine collagen of bones and skin.

Another reported advantage of HPMC capsules over gelatin capsules is related to the difference in moisture content of the shells. Because HPMC shells contain significantly less moisture compared to hard gelatin capsules by almost one third, it is compatible with hygroscopic materials. While HPMC shells physical strength tolerates wide range of environmental conditions, hard gelatin capsules readily becomes brittle and unusable in low humidity. One offered solution to this problem is to add PEG 4000 to the gelatin. As such the brittleness of the capsules will be minimized and encapsulation of hygroscopic materials becomes possible (19). Ciper and Bodmeier (69) have found that the addition of PEGs (400 or 1500 but not 4000) up to $5 \% \mathrm{w} / \mathrm{w}$ to the gelatin (Fastcaps) resulted in a significant disintegration time decrease in vitro (44 $\pm 6 \mathrm{~s})$ and in the mouth of four healthy volunteers $(13 \pm 4 \mathrm{~s})$ without affecting the mechanical properties of the capsules. Similar results were obtained when xylitol and sorbitol were used instead of PEGs. It is possible that the incompatibility of PEG and gelatin is why such capsules have not been produced in large scale.

The cross-linking of gelatin that affects product dissolution and disintegration has not been observed for HPMC capsules under similar conditions. This makes HPMC capsules compatible with wider range of products except for some oxidizing agent (70).

It may be expensive for the pharmaceutical industry to reformulate their products to make use of HPMC capsules as the benefits achieved might not be weighing out the cost. However, for new capsule products, HPMC capsules should become an option. Currently marketed products using HPMC capsules filled with herbal formulations benefit from flexible regulations over this category of supplements. Such regulations are expected to be tougher in the future which may lead to the number of HPMC capsule products to become static. In order to expand the use of HPMC capsules in new products, official bodies such as FDA should endorse their use and pharmacopeias should start to provide monographs regarding the specification and tests carried out for such capsules. With more interest exercised from the big pharmaceutical manufacturers to use HPMC capsules, definitely there will be greater number of products in the market.

\section{CONCLUSION}

The dominating capsule manufacturers are currently the Shionogi Qualicaps Co. Ltd. and Capsugel of Pfizer. Several published literature are from scientists affiliated to their own manufacturing companies and therefore may have overemphasized the potential of HPMC capsules to replace hard gelatin capsules. Two important areas where improvements have to be achieved in order to qualify the HPMC capsules ahead of gelatin capsules are in their machineability and in the in vitro and in vivo disintegration/dissolution performances. The main area where HPMC capsules can have better prospect compared to gelatin capsules is in wider patients' preferences and the dietary sensitivities in certain markets. 


\section{ACKNOWLEDGMENTS}

The author would like to thank Dr. Abedelnasser Abulrob who was very helpful in the literature survey.

\section{REFERENCES}

1. G.A. Burdock. Safety assessment of hydroxypropyl methylcellulose as a food ingredient. Food Chem Toxicol, 45:2341-51, 2007.

2. J.A. Ji, J. Liu, S.J. Shire, T.J. Kamerzell, S. Hong, K. Billeci, Y. Shen and Y.J. Wang. Characteristics of rhVEGF release from topical hydrogel formulations. Pharm Res, 27:644-54, 2010.

3. M. Akram, S.B. Shyum and S. Gauhar. Development of new ophthalmic suspension prednisolone acetate 1\%. Pak J Pharm Sci, 23:14954, 2010.

4. C.L. Li, L.G. Martini, J.L. Ford and M. Roberts. The use of hypromellose in oral drug delivery. J Pharm Pharmacol, 57:533-46, 2005.

5. A. Mesnukul, K. Yodkhum and T. Phaechamud. Solid dispersion matrix tablet comprising indomethacin-PEG-HPMC fabricated with fusion and mold technique. Indian J Pharm Sci, 71:413-20, 2009.

6. Shayeda, R. Gannu, C.R. Palem and Y.M. Rao. Development of novel bioadhesive buccal formulation of diltiazem: In vitro and in vivo characterization. PDA J Pharm Sci Technol, 63:4018, 2009.

7. Y. Rane, R. Mashru, M. Sankalia and J. Sankalia. Effect of hydrophilic swellable polymers on dissolution enhancement of carbamazepine solid dispersions studied using response surface methodology. AAPS PharmSciTech, 8:Article 27, 2007.

8. J. Varshosaz, N. Tavakoli and A. Serri. Preparation and in vitro characterization of piroxicam enteric coated pellets using powder layering technique. Pharm Dev Technol. 2009;14(3):305-11.

9. USP. Front Matter: Excipients. USP30 NF 25 (2007).

10. Robert O. Williams III, Matthew A. Sykora and Vorapann Mahaguna. Method to recover a lipophilic drug from hydroxypropyl methylcellulose matrix tablets. AAPS PharmSciTech, 2: Article 8, 2001.

11. Podczeck, F.; Jones, B.E., Pharmaceutical Capsules. Pharmaceutical Press, London, UK, 2004.

12. Jones, B., Hard Gelatin Capsules, in Aulton ME (ed), Pharmaceutics: The Science of Dosage Form Design. 2nd ed., Churchill Livingstone, Toronto, 2002.

13. Kendall, R.A.; Basit, A.W., The Role of Polymers in Solid Oral Dosage Forms, in Uchegbu IF and
Schätzlein AG (eds), Polymers in Drug Delivery. CRC Press Taylor and Francis Group, Florida, 2006.

14. L.A. Felton and G.S. Timmins. A nondestructive technique to determine the rate of oxygen permeation into solid dosage forms. Pharm Dev Technol, 11:141-7, 2006.

15. A.F. Gelb, C.F. Taylor, C. Cassino, C.M. Shinar, M.J. Schein and N. Zamel. Tiotropium induced bronchodilation and protection from dynamic hyperinflation is independent of extent of emphysema in COPD. Pulm Pharmacol Ther, 22:237-42, 2009.

16. O.P. Nmorsi, U.N. Kwandu and L.M. Ebiaguanye. Schistosoma haematobium and urinary tract pathogens co-infections in a rural community of Edo State, Nigeria. J Commun Dis, 39:85-90, 2007.

17. M.F. Gomes, M.A. Faiz, J.O. Gyapong, M. Warsame, T. Agbenyega, A. Babiker, F. Baiden, E.B. Yunus, F. Binka, C. Clerk, P. Folb, R. Hassan, M.A. Hossain, O. Kimbute, A. Kitua, S. Krishna, C. Makasi, N. Mensah, Z. Mrango, P. Olliaro, R. Peto, T.J. Peto, M.R. Rahman, I. Ribeiro, R. Samad, N.J. White; Study 13 Research Group. Pre-referral rectal artesunate to prevent death and disability in severe malaria: a placebo-controlled trial. Lancet, 373:55766, 2009.

18. M.S. Ku, W. Li, W. Dulina, F. Donahue, D. Cade, H. Benameur and K. Hutchisonc. Performance qualification of a new hypromellose capsule: Part I. Comparative evaluation of physical, mechanical and processability quality attributes of Vcaps Plus, Quali-V and gelatin capsules. Int J Pharm, 386:3041, 2010.

19. I. Chiwele, B.E. Jones, and F. Podczeck. The shell dissolution of various empty hard capsules. Chem Pharm Bull, 48: 951-956, 2000.

20. Augsburger, L.L., Hard and Soft Shell Capsules, in Banker GS and Rhodes CT (eds), Modern Pharmaceutics. Volume 121, Marcel Dekker, Inc, New York, 2002.

21. R.R. Grosswald, J.B. Anderson and C.S. Andrew (December, 1997). Method for the manufacture of pharmaceutical cellulose capsules. US Patent No. $5,698,155$.

22. R.R. Grosswald, J.B. Anderson and C.S. Andrew (May, 1998). Apparatus for the manufacture of pharmaceutical cellulose capsules. US Patent No. $5,750,157$.

23. R.R. Grosswald, J.B. Anderson and C.S. Andrew (May, 1998). Method for the manufacture of pharmaceutical cellulose capsules. US Patent No. $5,756,036$.

24. R.R. Grosswald, J.B. Anderson and C.S. Andrew (January, 2002). Method for the manufacture of 
pharmaceutical cellulose capsules. US Patent No. $6,337,045$.

25. T. Yamamoto, K. Abe and S. Matsuura (November, 1993). Hard capsule for pharmaceutical drugs and method for producing the same. US Patent No. 5,264,223.

26. T. Yamamoto, K. Abe and S. Matsuura (July, 1995). Hard capsule for pharmaceutical drugs and method for producing the same. US Patent No. 5,431,917.

27. T. Yamamoto, S. Matsuura, K. Hashimoto, K. Abe, K. Akal (April, 1999). Hard capsules. European Patent No. EP0592130B1.

28. J.H. Yang (June, 2002). Cellulose capsule using mixed solution of pectin and glycerin and the manufacturing process thereof. US Patent No. $6,410,050$.

29. D. Cade, R. Scott, X. He (February, 2003). Polymer film compositions for capsules. US Patent $6,517,865$.

30. C.M. Keary and W.A. Heeschen. Wet film dimensions of capsule walls during dip coating. Drug Dev Ind Pharm 28:1059-76, 2002.

31. M. Kuentz, B. Rothenhäusler and D. Röthlisberger. Time domain $1 \mathrm{H}$ NMR as a new method to monitor softening of gelatin and HPMC capsule shells. Drug Dev Ind Pharm, 32:1165-73, 2006.

32. S. Missaghi and R. Fassihi. Evaluation and comparison of physicomechanical characteristics of gelatin and hypromellose capsules. Drug Dev Ind Pharm, 32:829-38, 2006.

33. M. Ciper and R. Bodmeier. Modified conventional hard gelatin capsules as fast disintegrating dosage form in the oral cavity. Eur J Pharm Biopharm, 62:178-84, 2006

34. F. Cilurzo, F. Selmin, P. Minghetti, L. Montanari, C. Lenardi, F. Orsini and G. Poletti. Comparison between gamma and beta irradiation effects on hydroxypropylmethylcellulose and gelatin hard capsules. AAPS PharmSciTech, 6(4):E586-93, 2005.

35. N. Donauer and R. Löbenberg. A mini review of scientific and pharmacopeial requirements for the disintegration test. Int J Pharm, 345: 2-8, 2007.

36. Y. Wu, F. Zhao and M. Paborji. Effect of fill weight, capsule shell, and sinker design on the dissolution behavior of capsule formulations of a weak acid drug candidate BMS-309403. Pharm Dev Technol, 8:379-83, 2003.

37. S. Tochio, S. Nagata and S. Yamashita. The influence of the composition of test fluids on dissolution from HPMC capsules. AAPS PharmSci, 4(S1), abstract W4340, 2002.

38. O. Honkanen, P. Laaksonen, J. Marvola, S. Eerikäinen, R. Tuominen and M. Marvola. Bioavailability and in vitro oesophageal sticking tendency of hydroxypropyl methylcellulose capsule formulations and corresponding gelatine capsule formulations. Eur J Pharm Sci, 15:479-88, 2002.

39. Honkanen, O., Biopharmaceutical Evaluation of Orally and Rectally Administered Hard Hydroxypropyl Methylcellulose Capsules. Academic Dissertation, University of Helsinki, Finland, 2004.

40. E.T. Cole, R.A. Scott, D. Cade, A.L. Connor and I.R. Wilding. In vitro and in vivo pharmacoscintigraphic evaluation of ibuprofen hypromellose and gelatin capsules. Pharm Res, 21:793-8, 2004.

41. G.A. Digenis, T.B. Gold and V.P. Shah. Crosslinking of gelatin capsules and its relevance to their in vitro-in vivo performance. J Pharm Sci, 83:91521, 1994.

42. D. Stein and D.S. Bindra Stabilization of hard gelatin capsule shells filled with polyethylene glycol matrices. Pharm Dev Technol, 12:71-7, 2007.

43. L. Kalantzi, R. Page, E Nicolaides, G. Digenis and C. Reppas. In vitro methods can forecast the effects of intragastric residence on dosage form performance. Eur J Pharm Sci, 33:445-51, 2008.

44. T.A. Adesunloye and P.E. Stach. Effect of glycine/citric acid on the dissolution stability of hard gelatin capsules. Drug Dev Ind Pharm, 24:493-500, 1998.

45. M.M. Al-Tabakha and A.I. Arida. A study of the effect of jet milling process with or without pretreatment on aerosolisation characteristics of FITCdextran particles. J Med J, 40: 250-261, 2006.

46. M.M. Al-Tabakha and A.I. Arida. The effects of capsule fill weight and drug/carrier blend ratio on the aerosolization of a model drug from a Spinhaler. SPJ, 15:127-134, 2007.

47. J.C. Birchall, B.E. Jones, A. Morrissey and B.E. Jones. A comparison of the puncturing properties of gelatin and hypromellose capsules for use in dry powder inhalers. Drug Dev Ind Pharm, 34:870-6, 2008.

48. R A. Scott and E. T. Cole (August, 2006). Enteric and colonic delivery using HPMC capsules. US Patent 7,094,425.

49. N. Huyghebaert, A. Vermeire and J.P. Remon. Alternative method for enteric coating of HPMC capsules resulting in ready-to-use enteric-coated capsules. Eur J Pharm Sci, 21:617-23, 2004.

50. K. Dvorácková, M. Rabisková, J. Gajdziok, D. Vetchý, J. Muselík, J. Bernatoniene, M. Bajerová and P. Drottnerová. Coated capsules for drug targeting to proximal and distal part of human intestine. Acta Pol Pharm, 67:191-9, 2010.

51. E.T. Cole, R.A. Scott, A.L. Connor, I.R. Wilding, H.U. Petereit, C. Schminke, T. Beckert and D. Cadé. Enteric coated HPMC capsules designed to achieve intestinal targeting. Int J Pharm, 231:83-95, 2002. 
52. A.C. Perkins, C.G. Wilson, P.E. Blackshaw, R.M. Vincent, R.J. Dansereau, K.D. Juhlin, P.J. Bekker and R.C. Spiller. Impaired oesophageal transit of capsule versus tablet formulations in the elderly. Gut, 35:1363-7, 1994.

53. L. Zhang, D. Russell, B.R. Conway and H. Batchelor. Strategies and therapeutic opportunities for the delivery of drugs to the esophagus. Crit Rev Ther Drug Carrier Syst, 25:259-304, 2008.

54. H. Batchelor. Bioadhesive dosage forms for esophageal drug delivery. Pharm Res, 22:175-181, 2005.

55. L. McCargar, D. Crail, R. Dansereau, W. Myers and M. Lane. The in-vitro porcine adhesion model is not predictive of the esophageal transit of risedronate tablets in humans. Int J Pharm, 222:191-7, 2001.

56. E. Osmanoglou, I.R. Van Der Voort, K. Fach, O. Kosch, D. Bach, V. Hartmann, A. Strenzke, W. Weitschies, B. Wiedenmann, L. Trahms and H. Mönnikes. Oesophageal transport of solid dosage forms depends on body position, swallowing volume and pharyngeal propulsion velocity. Neurogastroenterol Motil, 16:547-56, 2004.

57. C. Tuleu, M.K. Khela, D.F. Evans, B.E. Jones, S. Nagata and AW. Basit. A scintigraphic investigation of the disintegration behaviour of capsules in fasting subjects: A comparison of hypromellose capsules containing carrageenan as a gelling agent and standard gelatin capsules. Eur J Pharm Sci, 30:251$55,2007$.

58. O. Honkanen, J. Marvola, H. Kanerva, K. Lindevall, M. Lipponen, T. Kekki, A. Ahonen and M. Marvola. Gamma scintigraphic evaluation of the fate of hydroxypropyl ethylcellulose capsules in the human gastrointestinal tract. Eur J Pharm Sci, 21:671-8, 2004.

59. C. Tuleu, A.W. Basit, W.A. Waddington, P.J. Ell and J.M. Newton. Colonic delivery of 4aminosalicylic acid using amylose-ethylcellulosecoated hydroxypropylmethylcellulose capsules. Aliment Pharmacol Ther, 16:1771-9, 2002.

60. O. Honkanen, H. Seppä, , S. Eerikäinen, R. Tuominen and M. Marvola. Bioavailability of ibuprofen from orally and rectally administered hydroxypropyl methylcellulose capsules compared to corresponding gelatine capsules. STP Pharma Sci, 11:181-185, 2001.

61. C. Tuleu, A.W. Basit, W.A. Waddington, P.J. Ell and J.M. Newton. Colonic delivery of 4aminosalicylic acid using amylose-ethylcellulosecoated hydroxypropylmethylcellulose capsules. Aliment Pharmacol Ther, 16:1771-9, 2002.

62. O. Honkanen, H. Seppä, , S. Eerikäinen, R. Tuominen and M. Marvola. Bioavailability of ibuprofen from orally and rectally administered hydroxypropyl methylcellulose capsules compared to corresponding gelatine capsules. STP Pharma Sci, 11:181-185, 2001.

63. F. Jamali and A. Aghazadeh-Habashi. Rapidly dissolving formulations for quick absorption during pain episodes: ibuprofen. Int J Clin Pharmacol Ther, 46:55-63, 2008.

64. A. Aghazadeh-Habashi and F. Jamali. Pharmacokinetics of meloxicam administered as regular and fast dissolving formulations to the rat: influence of gastrointestinal dysfunction on the relative bioavailability of two formulations. Eur J Pharm Biopharm, 70:889-94, 2008.

65. Y. El-Malah, S. Nazzal and C.B. Bottom. Hard gelatin and hypromellose (HPMC) capsules: estimation of rupture time by real-time dissolution spectroscopy. Drug Dev Ind Pharm, 33:27-34, 2007.

66. J.C. Leblanc, H. Yoon, A. Kombadjian and P. Verger. Nutritional intakes of vegetarian populations in France. Eur J Clin Nutr, 54:443-9, 2000.

67. E.H. Spencer, L.K. Elon and E. Frank. Personal and professional correlates of US medical students' vegetarianism. J Am Diet Assoc, 107:72-8, 2007.

68. J.H. Yang (December, 2007). Fish gelatin hard capsule and its preparation method. US Patent No. 7,309,499.

69. M. Ciper and R. Bodmeier. Preparation and characterization of novel fast disintegrating capsules (Fastcaps) for administration in the oral cavity. Int $\mathrm{J}$ Pharm, 303:62-71, 2005.

70. Y. Sakata and M. Otsuka. Evaluation of relationship between molecular behaviour and mechanical strength of pullulan films. Int J Pharm, 374:33-8, 2009. 